\title{
Concomitant leukocytosis and lymphopenia predict significant pathology at CT of acute abdomen: a case-control study
}

Alexandra Platon ${ }^{1}$, Chloe Frund ${ }^{1}$, Laura Meijers ${ }^{1}$, Thomas Perneger ${ }^{2}$, Elisabeth Andereggen ${ }^{3}$, Minerva Becker ${ }^{1}$, Alice Halfon Poletti ${ }^{3}$, Olivier T. Rutschmann ${ }^{3}$ and Pierre-Alexandre Poletti ${ }^{{ }^{*}}$

\begin{abstract}
Background: Acute abdominal pain accounts for about 10\% of emergency department visits and has progressively become the primary indication for $\mathrm{CT}$ scanning in most centers. The goal of our study is to identify biological or clinical variables able to predict or rule out significant pathology (conditions requiring urgent medical or surgical treatment) on abdominal $C T$ in patients presenting to an emergency department with acute abdominal pain.

Methods: This was a retrospective cohort study performed in the emergency department of an academic center with an annual census of 60'000 patients. One hundred and-nine consecutive patients presenting with an acute non-traumatic abdominal pain, not suspected of appendicitis or renal colic, during the first semester of 2013, who underwent an abdominal CT were included.

Two medical students, completing their last year of medical school, extracted the data from patients' electronic health record. Ambiguities in the formulations of clinical symptoms and signs in the patients' records were solved by consulting a board certified emergency physician. Nine clinical and biological variables were extracted: shock index, peritonism, abnormal bowel sounds, fever $\left(>38^{\circ} \mathrm{C}\right.$ ), intensity and duration of the pain, leukocytosis (white blood cell count $>11 \mathrm{G} / \mathrm{L}$ ), relative lymphopenia ( $<15 \%$ of total leukocytes), and C-reactive Protein (CRP). These variables were compared to the $\mathrm{CT}$ results (reference standard) to determine their ability to predict a significant pathology.
\end{abstract}

Results: Significant pathology was detected on CT in 71 (65\%) patients. Only leukocytosis (odds ratio 3.3, $p=0.008$ ) and relative lymphopenia (odds ratio $3.8, p=0.002$ ) were associated with significant pathology on $C T$. The joint presence of these two anomalies was strongly associated with significant pathology on $C T$ (odds ratio 8.2, $p=0.033$ ). Leukocytosis with relative lymphopenia had a specificity of $89 \%$ (33/37) and sensitivity of 48\% (33/69) for the detection of significant pathology on CT.

Conclusion: The high specificity of the association between leukocytosis and relative lymphopenia amongst the study population suggests that these parameters would be sufficient to justify an emergency CT. However, none of the parameters could be used to rule out a significant pathology.

Keywords: Acute abdominal pain, Computed tomography, Emergency, Leukocytosis, Relative lymphopenia

\footnotetext{
* Correspondence: pierre-alexandre.poletti@hcuge.ch

${ }^{1}$ Department of Radiology, University Hospital of Geneva, 4 rue

Gabrielle-Perret-Gentil, 1205 Geneva, Switzerland

Full list of author information is available at the end of the article
}

(c) The Author(s). 2019 Open Access This article is distributed under the terms of the Creative Commons Attribution 4.0 International License (http://creativecommons.org/licenses/by/4.0/), which permits unrestricted use, distribution, and reproduction in any medium, provided you give appropriate credit to the original author(s) and the source, provide a link to the Creative Commons license, and indicate if changes were made. The Creative Commons Public Domain Dedication waiver (http://creativecommons.org/publicdomain/zero/1.0/) applies to the data made available in this article, unless otherwise stated. 


\section{Background}

Acute abdominal pain accounts for about $10 \%$ of emergency department visits [1] and has progressively become the primary indication for CT scanning in most centers [2], due to the diagnostic accuracy of CT in most cases of acute abdominal pain [3, 4]. It has been previously shown that CT imaging results in a change in the management plan of more than $40 \%$ of cases when compared with clinical evaluation prior to CT [3].

However, the liberal use of $\mathrm{CT}$ in emergency departments raises the issue of potential overuse in acute abdominal pain cases, as it exposes patients to the risks associated with ionizing radiation, along with the injection of contrast medium [5]. Furthermore, a prospective, randomized study revealed that routine CT scanning for every patient presenting to the emergency department with abdominal pain is not cost effective [6] .

Though there are well-established guidelines regarding the indications for CT in cases of suspected cholecystitis [7], appendicitis [8] and renal colic [9], based on medical history, clinical and laboratory tests and, in some instances, ultrasound, the same cannot be said for other causes of acute abdominal pain $[4,10]$. Acute abdominal pain can have multiple etiologies, from the most benign (functional pains, constipation) to the most severe (bowel obstruction, hollow organ perforation, mesenteric ischemia, etc.), between which it is unfortunately not always easy to differentiate solely on the basis of medical history and clinical examination at admission [10-16]. The triage of patients with acute abdominal pain to CT is usually a long process, which risks delaying treatment and slowing patient flow in already overcrowded emergency department.

This study sought to analyze whether an association between clinical and biological variables, among examinations routinely performed on patients presenting with not well defined acute abdominal pain, could predict or rule out the presence of a significant intra-abdominal condition on $\mathrm{CT}$, namely one requiring urgent medical or surgical treatment.

\section{Methods}

\section{Study design and settings}

This retrospective study was approved by the institutional review board of our hospital (CER-14-020). As the study was based on a review of medical records, informed written consent was not required.

The study was undertaken in a university emergency department with an annual volume of around 61,000 adult patients ( $>16$ years), of which $8 \%$ (4900) presenting with abdominal pain.

\section{Patient selection}

For patients presenting with acute abdominal pain, the decision to perform a radiological examination is taken jointly by the resident physician and her/his supervisor, based on patient medical history, clinical examination and laboratory test results.

Our study included adult patients ( $>16$ year-old), presenting with not well defined non-traumatic acute abdominal pain for whom an intravenous contrast-enhanced CT scan was ordered as initial imaging examination at admission. Patients presenting with suspected renal colic, appendicitis, or cholecystitis were excluded from the study, given that they benefit from a standardized management, with routine low-dose CT scanning without injection of iodinated contrast medium for suspected renal colic or appendicitis [17, 18], or abdominal ultrasound for suspected cholecystitis [7].

\section{Data collection}

The study population was identified by two medical students (CF, LM), completing their last year of medical school, under supervision of a board certified radiologist, by electronic search of the radiology department database, using the keywords "acute abdominal pain", among all patients referred for $\mathrm{CT}$ in the emergency department across a period of 20 weeks, which corresponds to the rotation duration of a single team of emergency physicians in our center. During this period, a total of 2041 patients presented to the emergency department for acute abdominal pain. The electronic search identified 971 CT reports. The following exclusion criteria were applied: patients for whom CT was ordered for suspected appendicitis $(n=315)$, for detection of stones in the biliary tract after ultrasound $(n=3)$, for suspected renal colic $(n=332)$ and for trauma $(n=212)$.

A total of 109 consecutive patients met our inclusion criteria and formed the study population.

Nine clinical, hematological and biological parameters systematically tested at patients' admission were extracted from patients' files and transcribed in a dichotomous form (normal or abnormal). This extraction was performed by the same medical students who already identified the study population. Ambiguities in the formulations of clinical symptoms and signs in the patients' records were solved by consulting a board certified emergency physician (OTR). The extracted parameters are listed below (the thresholds for abnormality are indicated in parenthesis):

1) shock index (heart rate/systolic blood pressure $>0.7$ ), 2) fever $\left(\geq 38^{\circ} \mathrm{C}\right)$, 3) intense pain (visual analogue scale $\geq 7 / 10$ ), 4) long-lasting pain $(>6 \mathrm{~h}), 5$ ) peritonism (abdominal guarding and/or tenderness to palpation), 6) abnormal bowel sounds (metallic sounds, reduced/absence of sounds), 7) leukocytosis (leukocyte count $>11 \mathrm{G} / \mathrm{L}$ ), 8) relative lymphopenia (lymphocyte count $<15 \%$ of total leukocytes), and 9) elevated C-reactive protein (CRP) $(>11 \mathrm{mg} / \mathrm{l})$. 


\section{CT technique}

CT examinations were performed on a 64-row equipment (Discovery CT 750 HD, General Electric Company, Milwaukee, WI, USA) from lung base to pelvis. Patients received an intravenous bolus of $120 \mathrm{~mL}$ non-ionic contrast material (Iohexol, $300 \mathrm{mg} \mathrm{I} / \mathrm{mL}$, GE Healthcare AG, Opfikon, Switzerland), at a flow rate of $3.5 \mathrm{~mL} / \mathrm{sec}-$ ond using a power injector, with a delay of $60 \mathrm{~s}$ before initiating CT data acquisition, followed by a $30 \mathrm{~mL}$ saline flush at the same flow rate.

The following CT acquisition parameters were used: $64 \times 1.25 \mathrm{~mm}$ collimation, 0.9 pitch, 0.7 -s gantry rotation period, $120 \mathrm{kV}$ tube potential, automated tube current modulation, 2-1 $\mathrm{mm}$ reconstruction thickness, $40 \%$ adaptive statistical iterative reconstruction (ASIR).

\section{Reference standard}

The diagnosis on the final CT report, validated by the attending emergency radiologist on call, was considered reference standard. The CT diagnosis, in the frame of this study, was considered positive in the presence of a significant pathology. A significant pathology was defined prior to the beginning of the data collection in consensus by the attending physicians representing the units of emergency medicine (AHP, OTR), emergency surgery (EA) and emergency radiology (AP, PAP), as a condition which explained the abdominal pain and required medical or surgical treatment. A CT without pathological finding, or which showed a condition already known before admission and unrelated to the acute abdominal pain, was considered negative, as well as CT showing only signs of constipation.

\section{Statistical analysis}

The data were recorded and analyzed using a dedicated statistical software pack $\left(\right.$ IBM $^{\ominus}$ SPSS $^{\bullet}$ Statistics 22, IBM Corporation, USA). First, descriptive statistics were generated. Continuous values were expressed as mean \pm standard deviation. Associations of significant findings on CT at admission and clinical and biological variables were performed using the Chi-squared test for categorical variables and a T-test for the means. Logistic regression analysis was performed to identify the variables associated with an increased likelihood of significant findings on CT examinations. The level of statistical significance for all tests was defined as a $p$ value $<0.05$.

\section{Results}

Our study population consisted of 109 patients, 60 women and 49 men, with a mean age of 59 years (range 16-94, inter-quartile range (IQR) 46-78). There were 71 (65\%) positive CT examinations. The group of patients with a positive CT was made up of 38 (54\%) women and $33(46 \%)$ men, with a mean age of 57 (range 16-93, IQR
41-77). The group of patients with a negative CT was made up of 22 (58\%) women and $16(42 \%)$ men, with a mean age of 62 years (range 20-94, IQR 50-79).

The most commonly found pathologies on CT in the study population were: ileitis/colitis (of infectious, inflammatory or ischemic causes) $(n=25)$, small bowel obstruction $(n=10)$, pancreatitis $(n=6)$, and diverticulitis $(n=5)$. The distribution of diagnoses for the positive CT scans is recorded in Table 1.One case of appendicitis, one case of renal colic, 4 cases of pyelonephritis and 6 cases of pancreatitis were identified on CT; these diagnoses had not been suspected before the $\mathrm{CT}$ scan was ordered.

For each of the nine analyzed parameters, the available data from our study group in numbers (n) and percentage (\%) are as follows: shock index, $n=95$ (87\%); fever, $n=92(84 \%)$; pain intensity, $n=58(53 \%)$; pain duration, $n=103$ (94\%); abnormal bowel sounds, $n=81$ (74\%); peritonism, $n=105$ (96\%); leukocytosis, $n=109$ (100\%); relative lymphopenia, $n=106$ (97\%); and CRP, $n=107$ (98\%). Only non-missing data was analyzed.

\section{Comparison between clinical and laboratory findings and CT results (reference standard)}

Table 2 shows the demographic, clinical and biological characteristics of our study population.

No difference was observed between patients with and without significant findings on $\mathrm{CT}$ with regards to mean age, gender, presence of positive shock index, fever, presence of abdominal guarding, pain duration, and intensity,

Table 1 Significant diagnoses on abdominal computed tomography

\begin{tabular}{ll}
\hline CT diagnoses & 71 \\
\hline Colitis, enteritis, inflammatory bowel disease & $\mathrm{N}(\%)$ \\
Small bowel occlusion & $25(35)$ \\
Pancreatitis & $10(14)$ \\
Diverticulitis & $6(9)$ \\
Hollow viscus perforation & $5(7)$ \\
Digestive tumors & $4(6)$ \\
Biliary tract diseases & $4(6)$ \\
(cholangitis, choledocal obstruction) & $4(6)$ \\
Pyelonephritis & $4(6)$ \\
Intra-abdominal hemorrhage & $3(4)$ \\
Vascular pathologies & $2(3)$ \\
(aortic dissection, superior mesenteric artery stenosis) & $1(1)$ \\
Mesenteric adenitis & $1(1)$ \\
Hepatic abscess & $1(1)$ \\
Appendicitis & $1(1)$ \\
Ureteral calculus &
\end{tabular}

$N$ number 
Table 2 Baseline characteristics of patients with and without significant findings on emergency abdominal computed tomography

\begin{tabular}{|c|c|c|c|}
\hline Characteristic & $\begin{array}{l}\text { Positive } C T \\
N=71\end{array}$ & $\begin{array}{l}\text { Negative } C T \\
N=38\end{array}$ & $P$-value \\
\hline Mean age, years & 57 & 62 & 0.2 \\
\hline Sex (male), N (\%) & $33 / 71(46)$ & $16 / 38(42)$ & 0.4 \\
\hline $\begin{array}{l}\text { Positive shock index, N (\%) } \\
\text { Missing }\end{array}$ & $\begin{array}{l}5 / 62^{a}(8) \\
9\end{array}$ & $\begin{array}{l}2 / 33^{\mathrm{a}}(6) \\
5\end{array}$ & 1 \\
\hline $\begin{array}{l}\text { Fever } \geq 38^{\circ} \mathrm{C}, \mathrm{N}(\%) \\
\text { Missing }\end{array}$ & $\begin{array}{l}11 / 59^{\mathrm{a}}(18) \\
12\end{array}$ & $\begin{array}{l}4 / 33^{\mathrm{a}}(12) \\
5\end{array}$ & 0.56 \\
\hline $\begin{array}{l}\text { Abdominal guarding, N (\%) } \\
\text { Missing }\end{array}$ & $\begin{array}{l}24 / 68^{\mathrm{a}}(35) \\
3\end{array}$ & $\begin{array}{l}10 / 37^{\mathrm{a}}(27) \\
1\end{array}$ & 0.51 \\
\hline $\begin{array}{l}\text { Pain duration > 6 h, N (\%) } \\
\text { Missing }\end{array}$ & $\begin{array}{l}55 / 68^{\mathrm{a}}(81) \\
3\end{array}$ & $\begin{array}{l}30 / 35^{\mathrm{a}}(86) \\
3\end{array}$ & 0.6 \\
\hline $\begin{array}{l}\text { Pain intensity } \geq 7 / 10, N \text { (\%) } \\
\text { Missing }\end{array}$ & $\begin{array}{l}22 / 39^{\mathrm{a}}(56) \\
32\end{array}$ & $\begin{array}{l}13 / 19^{\mathrm{a}}(68) \\
19\end{array}$ & 0.4 \\
\hline $\begin{array}{l}\text { Abnormal bowel sounds, N (\%) } \\
\text { Missing }\end{array}$ & $\begin{array}{l}25 / 53^{\mathrm{a}}(47) \\
18\end{array}$ & $\begin{array}{l}19 / 28^{a}(68) \\
10\end{array}$ & 0.10 \\
\hline \multicolumn{4}{|l|}{ Elevated C-reactive protein, N (\%) } \\
\hline $\begin{array}{l}\geq 11 \\
\text { Missing }\end{array}$ & $\begin{array}{l}43 / 69^{\mathrm{a}}(62) \\
2\end{array}$ & $\begin{array}{l}22 / 38^{\mathrm{a}}(58) \\
0\end{array}$ & 0.68 \\
\hline $\begin{array}{l}\geq 20 \\
\text { Missing }\end{array}$ & $\begin{array}{l}35 / 69^{\mathrm{a}}(51) \\
2\end{array}$ & $\begin{array}{l}18 / 38^{\mathrm{a}}(47) \\
0\end{array}$ & 0.84 \\
\hline $\begin{array}{l}\geq 40 \\
\text { Missing }\end{array}$ & $\begin{array}{l}30 / 69^{\mathrm{a}}(43) \\
2\end{array}$ & $\begin{array}{l}11 / 38^{\mathrm{a}}(29) \\
0\end{array}$ & 0.15 \\
\hline $\begin{array}{l}\text { Leukocytosis, N (\%) } \\
\text { Missing }\end{array}$ & $\begin{array}{l}38 / 71(54) \\
0\end{array}$ & $\begin{array}{l}10 / 38(26) \\
0\end{array}$ & 0.008 \\
\hline $\begin{array}{l}\text { Relative lymphopenia, N (\%) } \\
\text { Missing }\end{array}$ & $\begin{array}{l}48 / 69^{\mathrm{a}}(70) \\
2\end{array}$ & $\begin{array}{l}14 / 37^{\mathrm{a}}(38) \\
1\end{array}$ & 0.002 \\
\hline $\begin{array}{l}\text { Association of leukocytosis \& } \\
\text { relative lymphopenia, N (\%) }\end{array}$ & $33 / 69^{a}(48)$ & $4 / 37^{\mathrm{a}}(11)$ & $\leq 0.001$ \\
\hline Missing & 2 & 1 & \\
\hline
\end{tabular}

presence of abnormal bowel sounds, or elevated CRP. CRP was analyzed using a cutoff value of $11 \mathrm{mg} / \mathrm{l}$, as well as a threshold value of the median $(20 \mathrm{mg} / \mathrm{l})$ and the double of the median $(40 \mathrm{mg} / \mathrm{l})$. None of these CRP thresholds were associated with significant findings on CT. Leukocytosis and relative lymphopenia were associated with positive CT examinations $(p=0.008$ and $p=0.002$, respectively). The simultaneous presence of leukocytosis and relative lymphopenia was also associated with a positive CT $(p<0.001)$. Multivariate analysis confirmed that only the simultaneous presence of leukocytosis and relative lymphopenia was significantly associated with positive CT findings (Table 3).

For leukocytosis, the sensitivity for predicting a positive CT was 53.5\% (38/71), specificity 73.7\% (28/38), positive predictive value (PPV) $79 \%$ (38/48), and negative predictive value (NPV) $45.9 \%$ (28/61). For relative
Table 3 Logistic regression modeling of positive computed tomography findings as a function of leukocytosis, relative lymphopenia, and an association of both parameters

\begin{tabular}{lll}
\hline & \multicolumn{2}{l}{ Multivariate analysis } \\
\cline { 2 - 2 } & $\begin{array}{l}\text { Odds ratio } \\
(95 \% \mathrm{Cl})\end{array}$ & $P$ value \\
\hline Leukocytosis & $0.67(0.15-2.8)$ & 0.58 \\
Relative lymphopenia & $1.5(0.52-4.2)$ & 0.45 \\
$\begin{array}{lll}\text { Association leukocytosis \& } \\
\text { relative lymphopenia }\end{array}$ & $8.25(1.19-57.5)$ & 0.033 \\
\hline Cl confidence interval & &
\end{tabular}

lymphopenia, these values were, respectively, $69.6 \%$ (48/ 69), $62.2 \%$ (23/37), $77.4 \%(48 / 62)$ and $47.7 \%$ (21/44). The simultaneous presence of leukocytosis and relative lymphopenia has a high specificity and PPV, both $89.2 \%$ (33/37), for predicting a significant pathology on CT. The sensitivity and NPV, however, are limited, with both 47.8\% (33/69) (Table 4).

\section{Discussion}

The aim of our study was to determine which of the clinical and laboratory variables obtained at patients' admission to an emergency department were predictive of the presence of significant pathology on CT. Our results show that the only variables meaningfully associated with the presence of a significant pathology on CT are leukocytosis and relative lymphopenia, and that the joint presence of these two anomalies may warrant an abdominal CT on its own merit. However, none of the parameters could be used to rule out a significant pathology.

Some studies have suggested that leukocytosis is associated with the presence of pathology on the CT of patients presenting for acute abdominal pain. Thus, in an Australian retrospective study conducted in 2006 by L. Modahl et al., the authors showed that the presence of leukocytosis was a good predictor of a positive CT, as well as the pediatric age and the presence of specified pre-CT diagnosis [19]. Leukocytosis was the only parameter analyzed in both this study and in ours. However, as no pediatric cases were included in our study, our results cannot be accurately compared to this latter study. A recent study indicated that leukocytosis, together with

Table 4 Sensitivity, specificity, PPV, and NPV for predicting a significant diagnosis on abdominal computed tomography

\begin{tabular}{lllll}
\hline & Sensitivity & Specificity & PPV & NPV \\
\hline Leukocytosis & 53.5 & 73.7 & 79.2 & 45.9 \\
& $(38 / 71)$ & $(28 / 38)$ & $(38 / 48)$ & $(28 / 61)$ \\
Relative lymphopenia & 69.6 & 62.2 & 77.4 & 47.7 \\
& $(48 / 69)$ & $(23 / 37)$ & $(48 / 62)$ & $(21 / 44)$ \\
Association of leukocytosis & 47.8 & 89.2 & 89.2 & 47.8 \\
\& relative lymphopenia & $(33 / 69)$ & $(33 / 37)$ & $(33 / 37)$ & $(33 / 69)$
\end{tabular}

PPV positive predictive value, $N P V$ negative predictive value 
CRP, is a good predictor of a positive CT [20], particularly in patients with right iliac fossa pain. Our study only partially confirms these results as it does effectively show an association between leukocytosis and the presence of abdominal pathology on CT, but it does not confirm the predictive role of CRP and patients with right iliac fossa pain were not included.

An association between relative lymphopenia and appendicitis has been reported in scientific literature [21, 22], but, to our knowledge, there have been no studies to directly correlate relative lymphopenia with CT results in patients presenting with abdominal pain where appendicitis was not suspected. Lymphopenia has, however, been reported as a marker of stress [23] and of infectious pathologies [24]. More broadly, it has been identified as a good predictor of bacteremia in emergency departments [25], and reported as better than CRP or leukocyte count [26]. Its presence has also been noted as a negative prognosis factor in surgical intensive care unit patients [24]. Based on these studies, it is not surprising that our analysis revealed an association between lymphopenia and significant pathology on CT.

CRP, on the other hand, was of no interest in the prediction of CT pathology, regardless of the threshold used in the frame of this study. Our results support those of a prospective study which noted no significant difference in the CRP values in patients with non-specific and non-severe abdominal pain compared with patients with severe abdominal pain [27]. Furthermore, CRP may stay low despite serious acute conditions if tested very early or if the patient has taken drugs with anti-inflammatory effects, such as non-steroidal anti-inflammatory drugs, corticosteroids, or antibiotics, before consultation [28, 29].

A meta-analysis has nonetheless shown that the combination of a CRP threshold of $>50$ and elevated leukocytosis $(>15 \mathrm{G} / \mathrm{L})$ had a high PPV for differentiating between emergency abdominal pathologies, requiring $\mathrm{CT}$, and those which are not emergencies. However, only $8.7 \%$ of patients in this study presented with these two variables [29], meaning that this criteria is not useful in a routine clinical setting.

The clinical variables analyzed were not predictive of the presence of significant pathology on CT. Several studies have also demonstrated the overall limitations of clinical variables in the evaluation of acute abdominal pain, with specificity often reported as less than $50 \%$ [12] and accuracy between 47 and 76\% [13-16]. However, it has been established that the value of clinical examinations varied between abdominal conditions, with clinical examinations reported as more effective with abdominal conditions in which pain is localized $[7-9,11,20]$, compared with widespread pain. Our study population did not include these suspected diagnoses, apart from diverticulitis, for which a care plan is usually put in place based on clinical examination. The patients in our study presented with less specific abdominal pain with a variety of causes, which explains the difficulty in finding common clinical signs among the different conditions found, and, by extension, the weakness of the clinical variables in the prediction of significant pathology on CT.

Similarly, some conditions found on CT, such as bowel obstruction, often do not cause fever. Others, such as diverticulitis, are not associated with abnormal bowel sounds. Furthermore, fever may not be present in older patients, or those who have taken antipyretic drugs. Pain with a duration of over $6 \mathrm{~h}$ before admission was also not relevant according to our analysis. This concurs with Eskelinen $\mathrm{M}$ et al.'s observations in a broad prospective study of 1333 cases, which evaluated, among others, how well pain duration predicted severity [30].

Combining leukocytosis and relative lymphopenia, we obtained a specificity of nearly $90 \%$ for the prediction of significant pathology on $\mathrm{CT}$. If $\mathrm{CT}$ was routinely ordered in the presence of this combination of signs, more than one third $(34 \%, 37 / 106)$ of the patients in our study could have benefited from accelerated care, without the need for other preliminary investigations or in-depth clinical examinations. Such a process has potential to improve patient flow, reduce morbidity and save medical resources.

On the other hand, due to a low sensitivity and negative predictive value (48\%), the absence of leukocytosis and lymphopenia does not indicate a lack of significant pathology on CT. The selection of patients with non-specific abdominal pain who do not require CT must continue to be based on the assessment by a clinician, by means of a series of clinical and laboratory tests $[11,30]$.

Our study has several limitations. First, it was a pilot study, focusing on a limited number of patients at a single hospital. It is also possible that variables which were not significant in our study could in fact be significant among a larger cohort of patients and could form the basis of a predictive score. However, the main aim of our study was to identify the variables which best predicted the presence of a serious pathology, which were already significant in a small group. In addition, due to its retrospective design, we were confronted with a certain amount of missing data, particularly with regards to patients' medical history and clinical examination.

Furthermore, our study population was subject to a possible selection bias, as it was made up of patients who had already been selected for CT scanning, that is, patients whose clinicians suspected significant abdominal pathology based on the examinations already undertaken. The number of patients with abdominal pain who were not referred for CT scanning, however, is not known; it is thus not possible to determine whether the criteria we have deemed predictive of significant pathology on CT, namely 
leukocytosis and relative lymphopenia, would still be significant without prior triage by a clinician. A prospective study on all patients presenting with abdominal pain, with systematic examination of these laboratory tests is therefore recommended to confirm the results of this pilot study.

\section{Conclusion}

In conclusion, the results of our preliminary study suggest that the association of leukocytosis and relative lymphopenia may predict the presence of significant pathology on CT, with a specificity and PPV close to $90 \%$. We recommend the undertaking of prospective studies to confirm these results, in which case the decision to order a CT scan could be accelerated in more than $30 \%$ of patients presenting with abdominal pain, not suspected of appendicitis or renal colic.

\section{Abbreviations}

CRP: C-reactive protein; CT: Computed tomography; IQR: Inter-quartile range; NPV: Negative predictive value; PPV: Positive predictive value; WBC: White blood cell

\section{Acknowledgements}

Not applicable

\section{Funding}

This research did not receive any specific grant from funding agencies in the public, commercial, or not-for-profit sectors.

\section{Availability of data and materials}

The datasets used and analyzed during the current study are available from the corresponding author on reasonable request

\section{Authors' contributions}

PAP and AP did conceive the study; PAP, AP, OTR, EA and MB did equally contribute to the design of the study; $C L, L M$ and AHP did collect the data; TP performed the statistical analysis of the data; AP, CF, LM, AHP, MB, TP, EA and OTR carried the data analysis and interpretation; all authors did participate to the drafting of the manuscript; PAP coordinated the whole study; all authors read and approved the final manuscript.

\section{Ethics approval and consent to participate}

This retrospective study was approved by the institutional review board of the Geneva University Hospital, Switzerland (CER-14-020). As the study was based on a review of medical records, informed written consent was not required.

\section{Consent for publication}

Not applicable

\section{Competing interests}

The authors declare that they have no competing interests.

\section{Publisher's Note}

Springer Nature remains neutral with regard to jurisdictional claims in published maps and institutional affiliations.

\section{Author details}

${ }^{1}$ Department of Radiology, University Hospital of Geneva, 4 rue Gabrielle-Perret-Gentil, 1205 Geneva, Switzerland. '2Division of Clinical Epidemiology, University Hospital of Geneva, 4 rue Gabrielle-Perret-Gentil, 1205 Geneva, Switzerland. ${ }^{3}$ Department of Community, Primary Care and Emergency Medicine, University Hospital of Geneva, 4 rue Gabrielle-Perret-Gentil, 1205 Geneva, Switzerland.
Received: 20 August 2018 Accepted: 11 January 2019

Published online: 18 January 2019

\section{References}

1. Excoffier S, Poletti PA, Brandstatter H. Acute abdominal pain of the upper abdomen: which imaging to choose? Rev Med Suisse. 2013;9:1710 17121714.

2. Larson DB, Johnson LW, Schnell BM, Salisbury SR, Forman HP. National trends in CT use in the emergency department: 1995-2007. Radiology. 2011; 258:164-73

3. Abujudeh $\mathrm{HH}$, Kaewlai R, McMahon PM, et al. Abdominopelvic CT increases diagnostic certainty and guides management decisions: a prospective investigation of 584 patients in a large academic medical center. AJR Am J Roentgenol. 2011;196:238-43.

4. Paolantonio $P$, Rengo $M$, Ferrari $R$, Laghi A. Multidetector $C T$ in emergency radiology: acute and generalized non-traumatic abdominal pain. $\mathrm{Br} J$ Radiol. 2016:89:20150859.

5. Brenner DJ, Hall EJ. Computed tomography--an increasing source of radiation exposure. N Engl J Med. 2007;357:2277-84.

6. Lehtimaki $\mathrm{T}$, Juvonen $\mathrm{P}$, Valtonen $\mathrm{H}$, Miettinen $\mathrm{P}$, Paajanen $\mathrm{H}$, Vanninen $\mathrm{R}$. Impact of routine contrast-enhanced $C T$ on costs and use of hospital resources in patients with acute abdomen. Results of a randomised clinical trial. Eur Radiol. 2013;23:2538-45.

7. Yarmish GM, Smith MP, Rosen MP, et al. ACR appropriateness criteria right upper quadrant pain. J Am Coll Radiol. 2014;11:316-22.

8. Smith MP, Katz DS, Lalani $T$, et al. ACR appropriateness criteria(R) right lower quadrant pain--suspected appendicitis. Ultrasound Q. 2015;31:85-91.

9. Bredemeyer M. ACR appropriateness criteria for acute onset of flank pain with suspicion of stone disease. Am Fam Physician. 2016;94:575-6.

10. Stoker J, van Randen A, Lameris W, Boermeester MA. Imaging patients with acute abdominal pain. Radiology. 2009;253:31-46.

11. Gans SL, Pols MA, Stoker J, Boermeester MA. Guideline for the diagnostic pathway in patients with acute abdominal pain. Dig Surg. 2015;32:23-31.

12. Lameris $W$, van Randen $A$, van Es HW, et al. Imaging strategies for detection of urgent conditions in patients with acute abdominal pain: diagnostic accuracy study. BMJ. 2009;338:b2431.

13. Ng CS, Watson CJ, Palmer CR, et al. Evaluation of early abdominopelvic computed tomography in patients with acute abdominal pain of unknown cause: prospective randomised study. BMJ. 2002:325:1387.

14. Rosen MP, Sands DZ, Longmaid HE 3rd, Reynolds KF, Wagner M, Raptopoulos V. Impact of abdominal CT on the management of patients presenting to the emergency department with acute abdominal pain. AJR Am J Roentgenol. 2000;174:1391-6.

15. Siewert B, Raptopoulos V, Mueller MF, Rosen MP, Steer M. Impact of CT on diagnosis and management of acute abdomen in patients initially treated without surgery. AJR Am J Roentgenol. 1997;168:173-8.

16. Taourel P, Baron MP, Pradel J, Fabre JM, Seneterre E, Bruel JM. Acute abdomen of unknown origin: impact of $C T$ on diagnosis and management. Gastrointest Radiol. 1992;17:287-91

17. Poletti PA, Platon A, Rutschmann OT, et al. Abdominal plain film in patients admitted with clinical suspicion of renal colic: should it be replaced by lowdose computed tomography? Urology. 2006;67:64-8.

18. Poletti PA, Platon A, De Perrot T, et al. Acute appendicitis: prospective evaluation of a diagnostic algorithm integrating ultrasound and low-dose CT to reduce the need of standard CT. Eur Radiol. 2011;21:2558-66.

19. Modahl L, Digumarthy SR, Rhea JT, Conn AK, Saini S, Lee SI. Emergency department abdominal computed tomography for nontraumatic abdomina pain: optimizing utilization. J Am Coll Radiol. 2006;3:860-6.

20. Ozan E, Atac GK, Evrin T, Alisar K, Sonmez LO, Alhan A. Do C-reactive protein level, white blood cell count, and pain location guide the selection of patients for computed tomography imaging in non-traumatic acute abdomen? Emerg Radiol. 2017;24:25-30.

21. Jahangiri M, Wyllie JH. Peripheral blood lymphopenia in gangrenous appendicitis. BMJ. 1990;301:215.

22. Devuyst $O$, Maldague $P$, Francois $P$, Dekeuleneer $R$, Michaux JL. Time-course of lymphopenia in gangrenous appendicitis. Lancet. 1991;338:1074.

23. Ramaekers LH, Theunissen PM, Went K. Acute lymphopenia, stress, and plasma cortisol. Arch Dis Child. 1975;50:555-8.

24. Grossbard $\amalg$, Desai MH, Lemeshow S, Teres D. Lymphocytopenia in the surgical intensive care unit patient. Am Surg. 1984;50:209-12. 
25. Wyllie DH, Bowler IC, Peto TE. Relation between lymphopenia and bacteraemia in UK adults with medical emergencies. J Clin Pathol. 2004;57:950-5.

26. de Jager CP, van Wijk PT, Mathoera RB, de Jongh-Leuvenink J, van der Poll T, Wever PC. Lymphocytopenia and neutrophil-lymphocyte count ratio predict bacteremia better than conventional infection markers in an emergency care unit. Crit Care. 2010;14:R192.

27. Salem TA, Molloy RG, O'Dwyer PJ. Prospective study on the role of Creactive protein (CRP) in patients with an acute abdomen. Ann R Coll Surg Engl. 2007;89:233-7.

28. Coyle JP, Brennan CR, Parfrey SF, et al. Is serum C-reactive protein a reliable predictor of abdomino-pelvic CT findings in the clinical setting of the nontraumatic acute abdomen? Emerg Radiol. 2012;19:455-62.

29. Gans SL, Atema JJ, Stoker J, Toorenvliet BR, Laurell H, Boermeester MA. Creactive protein and white blood cell count as triage test between urgent and nonurgent conditions in 2961 patients with acute abdominal pain. Medicine (Baltimore). 2015;94:e569.

30. Eskelinen $\mathrm{M}$, Lipponen P. Usefulness of history-taking in non-specific abdominal pain: a prospective study of 1333 patients with acute abdominal pain in Finland. In Vivo. 2012;26:335-9.

Ready to submit your research? Choose BMC and benefit from:

- fast, convenient online submission

- thorough peer review by experienced researchers in your field

- rapid publication on acceptance

- support for research data, including large and complex data types

- gold Open Access which fosters wider collaboration and increased citations

- maximum visibility for your research: over $100 \mathrm{M}$ website views per year

At BMC, research is always in progress.

Learn more biomedcentral.com/submissions 